in turn blossom and fragment again. The specialist in his narrow field may keep up with the factual information available, but the synthesis for the nonspecialist is becoming increasingly difficult. It is precisely this difficulty which justifies the series of new "advances" or "progress" publications, which for a while perform a very useful purpose.

This volume is the first of a series in the new branch of enzyme biochemistry: enzyme regulation. It constitutes the proceedings of a symposium on the regulation of enzyme activity. The symposium was wisely limited to consider one organ only and, therefore, all the articles refer to normal or neoplastic liver. The four sessions deal with the biochemistry of regulation of enzyme activity and synthesis, on the physiology and hormonal regulation, on the pathological physiology and drug effects, and finally on the regulation of enzyme activity in neoplasia. Each session consists of four to six lectures, usually ended by some comments from the session chairman and followed by a brief discussion on the most important parts of the session. The volume is closed by a special symposium lecture by $\mathrm{Sir}$ Hans Krebs.

The standard of papers is high, as would be expected by the illustrious list of experts attending the symposium. The information and references are up-to-date and the book is well produced and very well illustrated. It does not make easy reading for the non-expert, but it may be a very useful reference source for every biochemist or physiologist interested in enzyme function regulations of the liver.

\section{Repair from Genetic Radiation Damage and Differ- ential Radio Sensitivity}

Edited by F. H. Sobels. Pp. $x+454$, illustrated. Oxford, London, New York and Paris: Pergamon Press. 1963. 5 gns.

This book comprises the proceedings of a Symposium held at the University of Leiden in 1962, and as such it reflects much modern thought on the genetical effects of radiation. The material is, of course, very highly specialised, but for those with a particular interest in this vastly important field it will prove invaluable. The subjects discussed by a most eminent group of participants range from the primary molecular lesion ultimately responsible for mutagenesis, through many aspects of the biology of genetic radiation damage to post-radiation procedures which modify the radiation response. A great deal of space is devoted to the most interesting finding of differential radiosensitivity at various stages of oogenesis and spermatogenesis, and also to discussions of the effects of varying radiation dose rates and fractionation procedures; in these fields the contributions of F. H. Sobels, W. L. Russell and C. E. Purdom may perhaps be mentioned as being particularly interesting.
The majority of the material presented in the book is of necessity highly technical, but the presentation is clear throughout and the standard of production of the book is also excellent.

Lysergic Acid (LSD 25) and Ritalin in the Treatment?
of Neurosis

Thomas M. Ling and John Buchman. Pp. 172흠 Sidcup, Kent: Lambard Press. 1963. 21s.

Lysergic acid diethylamide (LSD) is a poten $\mathbb{D}$ hallucinogenic drug which has been reported to bญ्? helpful in the psychotherapy of a number of disorders such as obsessional states, personality dise orders, psychopathy, various neuroses etc. Its main advantage is that it facilitates the recall of early memories with their associated emotions and en? ables the patient to abreact his earlier experiences and sometimes to re-enact them as if they were actually present.

This book is mainly an account of a series of patients treated by lysergic acid and ritalin which is used to potentiate its effects. The authors describqv the treatment of a series of 350 patients, but giveno clinical details of these and no attempt is made to analyse or control the results. If this had beent done the book could have been of much greate value.

Arterial Surgery: Proceedings of a Conference held $\mathbb{D}$ at Law Hospital, Carluke, Scotland. January, 1963를 Edited by W. A. Mackey, J. A. MacFarlañ M. S. Christian. Pp. ix +170 , illustrated. Oxford, London, New York and Paris : Perganento Press. 1964. 50s.

Arterial surgery is still a new field of endeavour which ideas are constantly changing and therefore ao balanced symposium is of great value in reviewing current opinions. This book performs the task admir $-\bar{O}$ ably in that it serves as a short review covering then whole field, from the pathology to the treatment of such specialized conditions as mesenteric vascularo occlusion and coronary artery disease. A short list of references is provided at the end of each chapter

A major part of the discussion is taken up with the indications for arterial surgery. On the whole the speakers advocated a more cautious approach. than might have appeared in such a symposium giveno five years ago, but there is no doubt from the figures given that reconstructive surgery performed; on the correct indications gives excellent results.

The title of the book, 'Arterial Surgery' suggests. that its main value would be for surgeons, howevero the inclusion of chapters on carotid and renal artery stenosis, intracranial aneurysms and anticoagulantso make it a book which will appeal to a wide audience? of postgraduate students. 